Ljubljana

\title{
ZUM BEGRIFF DER KOMMUNIKATIVEN FUNKTION IN DER LINGUISTIK
}

In diesem Artikel soll der Funktionsbegriff in der Linguistik diskutiert werden, insbesondere der Begriff der kommunikativen Funktion. Es geht nur um eine allgemeine Behandlung von Charakteristiken des Begriffs kommunikative Funktion. Am Anfang wird eine Reihe bekannter und doch recht unterschiedlicher Bedeutungsinterpretationen des Funktionsbegriffs in der Linguistik übersichtlich angeführt. Das zweite Unterkapitel versucht es, sich mit verschiedenen Gesichtspunkten der nach Wilhelm SCHMIDT definierten kommunikativen Funktion auseinanderzusetzen: $u$. a. mit ihrer Verwurzelung in der funktional-kommunikativen Sprachbeschreibung, mit dem Unterscheiden von kommunikativen Funktionen auf verschiedenen Ebenen (kommunikative Funktion des Textes, kommunikative Funktion der Ausdrucksmittel, kommunikative Funktion der Sprache bzw. des Sprachsystems), ferner zieht es eine hypothetisch annehmbare rezeptive Komponente der kommunikativen Funktion heran und macht schließlich den Versuch einer Distinktion zwischen direkter (unmittelbarer) und indirekter (mittelbarer) Ausprägung einzelner kommunikativer Funktionen. Es wird auch auf die Funktion verwiesen, die der Text für den Rezipienten hat. Der Abschluß bringt zusammenfassend die wesentlichen Erkenntnisse dieses Beitrags.

\section{ZUR PROBLEMATISIERUNG DES BEGRIFFS FUNKTION IN DER LINGUISTIK}

Die Verwendung des Funktionsbegriffs ist in der Linguistik problematisch insofern, als er von verschiedenen Autoren in unterschiedlichen Zussamenhängen gebraucht wird, was zu divergierenden Interpretationen der Begriffsbedeutung führt. Das ist u. a. auch Gegenstand der Beiträge von W. SCHMIDT (1982) und G. HELBIG (1968). Nach SCHMIDT (1982, passim) läßt sich in Übereinstimmung mit den Wörterbucherklärungen des Funktionsbegriffs (Funktion sei gleich 1. Aufgabe..., 2. gesetzmäßige Abhängigkeit..., WDG 2, 1967, S. 1424) die Verwendung des Funktionsbegriffs bei verschiedenen Linguisten und linguistischen Schulen in den folgenden Umrissen verfolgen:

1. Für die Kopenhagener Schule ist die Funktion die wechselseitige innere Abhängigkeit, Dependenz zwischen zwei Funktiven... als den Schnittpunkten wechselseitiger Beziehungen innerhalb des Sprachsystems.

2. Für die generative Transformationsgrammatik sind Funktionen Relationen zwischen Paaren syntaktischer Kategorien in der Tiefenstruktur. 
3. Amerikanische Deskriptivisten verstehen unter Funktionen die Positionen, in denen eine Form vorkommt.

4. Viele sprachwissenschaftliche Schulen fassen die Funktion als Aufgabe auf, als Leistung einer sprachlichen Erscheinung (sei es einzelner Sprachelemente oder des sprachlichen Systems, sei es einer Äußerung oder der Sprachtätigkeit überhaupt).

5. Die funktional ausgerichtete Prager Schule unterscheidet zwischen 2 Sprachfunktionen: zwischen kommunkativer und mentaler (diese entspricht im wesentlichen der traditionellen kognitiven Funktion). Die Prager Schule beachtet somit neben der "langue" auch die Ebene der "parole" und spricht auch über Funktionen einzelner Sprachmittel.

6. Von der sowjetischen Sprachwissenschaft wird der Funktionsbegriff manchmal mit Inhalt bzw. Bedeutung gleichgesetzt und somit den Kategorien Form und Struktur gegenübergestellt.

7. Gerhard HELBIG hebt den Untershied zwischen verschiedenen Funktionsebenen hervor und definiert die Funktion, wie folgt: "Unter Funktion verstehen wir folglich sämtliche Ebenen zwischen der Sprachstruktur und der Wirklichkeit, unter Inhalt (als Spezialfall der Funktion) nur die designative Funktion“ (1968, S. 283).

8. Die Textlinguistik unterscheidet allein auf der Textebene eine große Anzahl von Textfunktionen.

9. KAINZ kennt eine dialogische (intersubjektive) Funktion und eine monologische (intrasubjektive) Funktion der Sprache. Im Verhältnis zu den traditionellen Sprachfunktionen - der kognitiven und kommunikativen Funktion - sei nicht nur die monologische, sondern mitunter auch die dialogische Funktion kognitiv (Fachdiskussionen u. a. m.).

10. HARTUNG erörtert vier Grundfunktionen der Kommunikation, die generell Funktionen der äußeren Sprachtätigkeit erfassen:

- kommunikative Funktion (im traditionellen Sinne)

- Funktion als Träger der theoretisch-erkennenden Tätigkeit der Menschen (deckt sich aber nur zu einem Teil mit der traditionellen kognitiven Funktion)

- phatische oder Kontaktfunktion

- Entlastung oder Stabilisierung des 'psychischen Haushaltes' des Sprechers (19.76, S. 302).

Damit sind freilich nicht alle Varianten der Funktionsdeutungen in der Linguistik erschöpft, eine vollständige Übersicht konnte in diesem Rahmen nicht angestrebt werden. 
Offenbar muß von Fall zu Fall gesondert bestimmt werden, worauf der Funktionsbegriff überhaupt Bezug nimmt. Dabei ist in den folgenden Fällen die Möglichkeit gegeben, über die Funktion zu reden:

a) bei einzelnen Spracherscheinungen bzw. -formen (-elementen) als Bestandteilen des sprachlichen Systems;

b) bei der Sprache als dem Inbegriff für das Sprachsystem;

c) bei der Kommunikation (dem Kommunikationsereignis) als dem wechselseitigen (intersubjektiven) Interaktionsprozeß zwischen mehreren Subjekten, die sich zum Zweck des Kommunizierens des Sprachsystems bedienen;

d) beim Text als der resultativen Erscheinungskomponente des Kommunikationsprozesses.

\section{ZUR DEFINITION DES BEGRIFFES DER KOMMUNIKATIVEN FUNKTION}

Nach den Prinzipien der funktionalen Grammatik wurde die Funktion zunächt mit Bedeutung gleichgesetzt, später aber als intendierter und meist auch erreichter kommunikativer Effekt definiert. Der funktional-kommunikativen Sprachbeschreibung (SCHMIDT 1981) liegt ein komplizierter, jedoch real existierender Mechanismus von Beziehungen zwischen sprachlichem System und sprachlicher Tätigkeit zugrunde, wobei die Grundforderung die ist, "daß die funktional-kommunikative Sprachbeschreibung die Dialektik von sprachlich-kommunikativer Tätigkeit und Sprachsystem gibt ...“ (SCHMIDT 1982 a, S. 4). Sprachliche Kommunikation ist dabei verstanden als eine spezifische Art der menschlichen Tätigkeit, die aus kleineren, elementareren konstitutiven Einheiten - Handlungen und Operationen - besteht. Die Sprache bzw. das Sprachsystem ist Mittel, Instrument dieser Kommunikation, das Ergebnis und Voraussetzung der sprachlichen Tätigkeit zugleich (SCHMIDT 1982, 16), denn "Das Sein und das Werden einer Sprache ist durch die kommunikative Tätigkeit bedingt" (LANGNER 1982, S. 214). Da für menschliche Tätigkeit jeder Art ein mehr oder weniger konstantes Verhältnis von objektiven Gegebenheiten (Anforderungen, Aufgaben, Anregungen), subjektivem Wollen (Motivation, Absicht), von der Zielgerichtetheit der Tätigkeit und von der Art und Weise der Durchführung des Geplanten (im Rahmen gewisser Bedingungen) eine Rolle spielt, ist dasselbe Relationsgefüge auch für die zwischenmenschliche Kommunikation als einer besonderen Art geistiger Tätigkeit anzunehmen. Von einer solchen gegenseitigen Abhängigkeit verschiedener Parameter geht unter besonderer Berücksichtigung der intentionalen Komponente auch die SCHMIDTsche Definition der kommunikativen Funktion aus:

“Die Zielgerichtetheit von Tätigkeiten und ihrer konkreten Realisationen, nämlich der Handlungen mit ihren Operationen, und zugleich die Zweckbestimmtheit der Instrumente/Mittel von Tätigkeiten, Handlungen und Operationen nennen wir ihre Funktion" (SCHMIDT 1982, S. 15 f.). 
Da es dabei um die kommunikative Tätigkeit geht und um die Sprache als Mittel/Instrument dieser Tätigkeit, wird die Funktion eben als kommunikative Funktion bezeichnet.

Wir sehen, daß diese Definition zwei grundlegende Ebenen der kommunikativen Funktion berührt: einmal tritt in den Vordergrund die Tätigkeit, also Operationen, der Kommunikationsprozeß bzw. der Text als dessen Resultat, zum anderen die sprachlichen Mittel als Instrument der Tätigkeit. Im ersten Fall wird das Endziel einer kommunikativen Tätigkeit erfragt, das sog. Oberziel der Sprachhandlung (DIMTER 1981, S. 65), so daß kommunikative Funktion eines Resultats kommunikativer Tätigkeit (bzw. eines Textes) gewissermaßen die in dieser kommunikativen Tätigkeit enkodierte kommunikative Absicht ist. Oder mit Worten von DIMTER: "Die Funktion ... eines Textes ist, einem Sprecher zur Erreichung eines oder mehrerer Ziele zu dienen“ (1981, S. '85).

Im zweiten Fall haben wir es mit der Zweckbestimmtheit der Verwendung von sprachlichen Mitteln zu tun (nach DIMTER ebd., S. 65, "instrumentales Ziel"). Diese Zweckbestimmtheit der Verwendung von sprachlichen Mitteln ist antizipiert im Endziel des gesamten Kommunikationprozesses, ist ja die Realisierung dieses Endziels eines Kommunikationsereignisses durch einen zweckbestimmten Ausdrucksmitteleinsatz bedingt. Der Zielbegriff ist nach SCHMIDT (1982, S. 15) folglich in Zusammenhang zu bringen mit der gesamten Sprachtätigkeit, mit dem Kommunikationsprozeß, dem Text als dessen Ergebnis, ungeachtet der für die Realisierung dieses Kommunikationsprozesses gewählten Ausdrucksmittel, während der Zweck nach SCHMIDT (ebd.) konsequent mit dem strategischen (operationellen) Gesichtspunkt der Realisierúng des Kommunikationsprozesses einhergeht.

In Anlehnung an HANNAPPEL/MELENK (1979), die nach Worten MICHELS "klar zwischen Ziel und Weg. bzw. Ziel und Mittel unterscheiden" (MICHEL 1983, S. 469), könnte nach unserem Dafürhalten eine strenge Unterscheidung zwischen dem Zielcharakter der kommunikativen Funktion von kommunikativen Handlungen und dem Zweckcharakter der kommunikativen Funktion von Ausdrucksmitteln dahingehend relativiert werden, als genau genommen auch der Zielgerichtetheit von Tätigkeiten, Handlungen und Operationen i. w. S. ein allgemeiner Instrumentalcharakter zuzuschreiben ist, denn Handlungen sind letzten Endes nichts anderes als Wege (Mittel), die im Rahmen einer übergeordneten Tätigkeit (z. B. Kunst, Journalistik) zu gewissen Zielen führen.?

Den neusten Studien über die kommunikative Funktion zufolge (u. a. MICHEL 1986, HEUSINGER 1987, HEUSINGER 1987a) kann der ursprüngliche SCHMIDTsche Funktionsbegriff mit Definitionscharakter erweitert werden. So wird neuerdings im Zussamenhang mit dem Text von seiner Zweckbestimmtheit gesprochen in

1 Das Ziel der Handlung könnte somit u. U. erst die (erwünschte) Reaktion des Kommunikationspartners (im Sinne von Perlokution) sein. 
der Relation “Ziel - Bedingungen - Mittel - Zweck“ (MICHEL a.a.O., S. 67), vor allem unter den Aspekten: Welches Ziel unter welchen Bedingungen (z. B. Bedingungen der übergeordneten Tätigkeit) soll mit der Äußerung erreicht werden? Welche Absicht verfolgt der Autor mit seinem Text? Welchem Zweck dient der Text - aus der Sicht des Sprechers/Schreibers (im weiteren S/S) - im Rahmen einer spezifischen Tätigkeit des S/S (z. B. der Journalistik)?

Angesichts der oben übernommenen Definition der kommunikativen Funktion nach SCHMIDT (s. oben) drängen sich u. E zwei weiterführende Gedanken hypothetischer Natur auf, und zwar hinsichtlich der Existenz einer rezeptiven Komponente im Begriff der kommunikativen Funktion sowie hinsichtlich der Mittelbarkeit (indirekter Ausprägung) bzw. 'Unmittelbarkeit (direkter Ausprägung) von kommunikativen Funktionen. Bei aller Genauigkeit und Adäquatheit der oben erwähnten und ansonsten akzeptierten SCHMIDTschen Definition der kommunikativen Funktion scheint uns ihre Zukunftsbezogenheit, ihre "Zielgerichtetheit" ${ }^{\text {" }}$ bzw. ihre

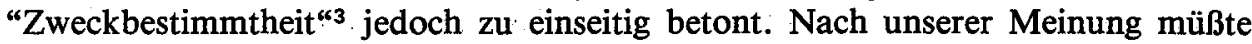
man im Kommunikationsprozeß noch einer weiteren Komponente Rechnung tragen, denn der Kommunikationsproze $\beta$ als im Prinzip vorprogrammierte Handlung, zugleich aber auch die Verwendung einzelner Ausdrucksmittel im Rahmen gewisser Operationen vermögen es, beim Empfänger zusätzliche und unvorhergesehene kommunikative Nebeneffekte ${ }^{4}$ herzvorzurufen. Die Folge ist, daß das erwünschte und vorprogrammierte Ziel bzw. die kommunikative Absicht ${ }^{5}$ einerseits und das erreichte (erwünschte oder unerwünschte) Ziel bzw. Resultat bzw. die Wirkung auf den Adressaten andererseits sich nicht immer gänzlich decken müssen, weil der Autor nicht in der Lage sein kann, die Wirkung der verwendeten Mittel völlig voraussehen und ihre potentiellen komplexen Möglichkeiten erfassen zu können. ${ }^{6}$ Man muß unterscheiden zwischen der kodierten Textfunktion (S/S) und der Funktion des Textes für den Rezipienten. Die kodierte Funktion (Textfunktion) wird in der Regel vom Rezipienten - wenn auch nicht gänzlich im Hinblick auf anteilige Mittel — dekodiert. In der Tat können (was häufig der Fall ist) beabsichtigte und realisierte Wirkung auseinanderfallen. Jede kommunikative Situation ist eine einmalige und praktisch unwiederholbare Kombination verschiedener Faktoren und Bedingungen mit auch unerwarteten, zufälligen, überraschenden Nachwirkungen. Es stimmt zwar,

2 "Ziel wird... verstanden als gedanklich vorweggenommener zukünftiger Zustand bzw. relativer Endpunkt einer Entwicklung, der aus einem Feld objektiver Möglichkeiten vom Menschen auf Grund einer Entscheidung bewußt ausgewähit... und festgelegt... wird und nur durch aktives Handeln der Menschen verwirklicht werden kann" (SCHMIDT 1982, S. 15).

3 "Der Zweck... ist an die Absicht des Menschen gebunden, bestimmte Mittel einzusetzen bzw. bestimmte Handlungen auszuführen, die der Erreichung seiner Ziele dienen... Der Zweck bestimmt sich... als das Ziel, um dessentwillen ein bestimmter Gegenstand gebraucht oder eine bestimmte Handlung ausgeführt wird“ (ebd.). (Beide Definitionen in Übereinstimmung mit dem Philosophischen Wörterbuch 1976.)

4 S. auch Genaueres darüber weiter unten.

5 . "Die Kommunikationsabsicht ist durch das Handlungsziel bestimmt, das mit der Kommunikationsaufgabe gegeben ist; sie ist das durch den Textproduzenten (subjektiv) angeeignete Kommunikationsziel." (SCHMIDT 1982, S. 16).

6 Vgl. Anm. 11. 
daß die meisten dieser Kombinationen (gegenseitiger Einwirkungen) gesellschaftlich konventionalisiert und gefestigt und somit normiert sind, allerdings sollten auch Abweichungen vom Konzipierten, sollte auch Unerwartetes als Komponente der kommunikativen Funktion von Kommunikationsprozessen bzw. von Ausdrucksmitteln beachtet werden. Wenn wir die Kommunikation als tätigkeitsbezogene Interaktion auffassen, so ist der Begriff der Absicht eigentlich als erstes Element, als Einleitepol eines ambivalenten, dialektischen Spannungsgefüges "AbsichtWirkung" zu betrachten. Dabei ist - wie oben ausgeführt - eine Übereinstimmung oder Nichtübereinstimmung beider Bestandteile (entgegengesetzter Pole) möglich, und dies führt zu verschiedenen Konsequenzen (s. unten). Eine Teilwirking des Textes $^{7}$ oder der Ausdrucksmittel muß also nicht unbedingt mit der Ausgangsabsicht übereinstimmen und kann sich $u$. U. einer bewußten Kontrolle entziehen. Der Effekt kann der Absicht mitunter sogar vorgreifen, wenn es sich um eine ausdrücklich spontane Ausdrucksweise handelt: Manches wirkt dagegen aufgrund der gegenseitigen Einwirkungen verschiedener Faktoren unbewußt erst später nach, und das ist manchmal nur schwer vorauszusehen. Eben diese unvorhergesehenen Auswirkungen in der geplanten Kommunikation als einer Ganzheit sowie beim Gebrauch einzelner Ausdrucksmittel zur Realisation des Kommunikationsprozesses - wir bezeichnen das mit dem Begriff resultative (rezeptive) Komponente der kommunikativen Funktion - sind u. E. eine der Triebkräfte der funktionsmäßigen Entfaltung des Sprachsystems und der Vervollständigung der sprachlichen Kommunikation. Käme es nicht zu diesen spontanen, auf inneren Gesetzmäßigkeiten beruhenden Verschiebungen, so führte das Stereotype an der Kommunikation zur Monotonie und Einengung der Ausdrucksmöglichkeiten. Aufgabe des Linguisten ist es, diese Veränderungen zu beobachten, sie zu registrieren, systematisieren und ihnen nach Bedarf das bestehende Sprachsystem anzupassen sowie die Veränderungen als Folgen eines automatisch verlaufenden Mechanismus auch anders allseitig zu bearbeiten. Somit berühren wir auch schon eine zweite, damit verwandte, jedoch nicht auch identische Frage, und zwar die der Mittelbarkeit bzw. Unmittelbarkeit der kommunikativen Funktionen. Auch hier geht es im Grunde um das Verhältnis zwischen kommunikativer Absicht und kommunikativer. Wirkung auf den Adressaten ${ }^{8}$, allerdings haben

7 Bei DIMTER (1981) beruhen funktional begründete Teiltexte auf parataktischen und hypotaktischen Beziehungen der Ziele im Text. (Nach THIELE 1983, S. 100).

8 Es handelt sich eigentlich um eine Dichotomie, die sich in verschiedenen Formen und Ausprägungen auf verschiedenen Ebenen der Sprachbeschreibung und Kommunikation betrachten läßt, von den einfachen Formen ausgehend, wo wir im Zusammenhang mit einzelnen Lexemen von ihrer Mehrdeutigkeit reden, von ihrer wörtlichen und übertragenen Bedeutung. So wie bei einzelnen Lexemen ist auch bei einzelnen Äußerungen und letztlich bei den Texten der Kontext der entscheidende Faktor, der zur Identifizierung der wirklich gemeinten Bedeutung des sprachlichen Zeichens beiträgt. (Ein Vergleich wäre auch mit den CHOMSKYschen Begriffen "competence“ - "performance“ zu ziehen.) Während bei einzelnen Lexemen diese Dichotomie vorwiegend in der regelmäßigen systemhaften Opposition zwischen Möglichkeit (Potenz) und Realisation (langue:parole) liegt, ist im breiteren kommunikativen Rahmen die Pole solcher Dichotomie auch im Verhältnis zwischen den Vorstellungen des Senders und der Rezipientenwelt zu suchen. Hierbei spielt das gesamte Spektrum von Determinanten des Kommunikationsprozesses eine Rolle, weil ein und dieselbe Mitteilung allein von verschiedenen Rezipienten unterschiedlich aufgenommen wird. MICHEL schreibt in diesem Zusammenhang: “... wird in aller Deutlichkeit noch einmal bestätigt, daß die Wirkung von Texten letztlich nicht von den Texten selbst, sondern von den soziologich-psychologischen Voraussetzungen des Textrezipienten. abhängt." 
wir dabei in erster Linie jene versteckten Einwirkungen auf den Adressaten ${ }^{9}$ vor Augen, die sich aus verschiedenen Gründen einer unmittelbaren Analyse der kommunikativen Funktion entziehen.

Der Sender versucht, im Text absichtlich doppelte kommunikative Absicht zu kodieren, bzw. er versucht vielmehr, seine wahre kommunikative Absicht durch verschiedene Mittel zu verschleiern, indem die eine (scheinbare) kommunikative Absicht direkt und sofort erkennbar sein sollte, die andere (tatsächliche) kommunikative Absicht, der "Textsinn" (BIEBERLE 1987, S. 199), jedoch durch bewußte Täuschung nach Möglichkeit unidentifiziert bleiben möge, obwohl sie im konkreten Text $^{10}$ die kommunikative Hauptrolle zu erfüllen hat. Auch auf der Ebene des Ausdrucksmittelarsenals (das trifft weitestgehend auch für die Kommunikationsverfahren zu) läßt sich analog dazu eine Art Dichotomie bei der zweckbestimmten Verwendung von einzelnen Ausdruckmitteln (auch von Kommunikationsverfahren) erkennen. ${ }^{11}$ Damit in Zusammenhang zu bringen ist die zentrale Rolle des strategischen Vorgehens (der operationale Gesichtspunkt) bei der Realisierung von Kommunikationsabsichten. Bei der Vertextung von Kommunikationsabsichten können somit verschiedene Kombinationen des Sichtbaren und Versteckten (des Transparenten und Untransparenten) zum Vorschein kommen, wobei sich gewöhnlich auch der Grad der Aufrichtigkeit und Ehrlichkeit seitens des Textproduzenten ändert: von dem lediglichen Bestreben, größere Wirksamkeit zu erzielen - sei es in alltäglichen Gebrauchstexten des wirtschaftlichen (Werbung) oder politischen Lebens (Agitation und Propaganda), bis hin zu regelrechtem Manipulieren mit dem Adressaten. ${ }^{12}$

Wenn jemand eine Person (übertrieben) lobt, kann er sie damit in der Tat indirekt kritisieren (wenn z. B. jemandem bei seinem Bewerben um eine neue Stelle Empfehlungen geschrieben werden). Wenn ein Sender es beabsichtigt, den Adressaten über etwas zu informieren, so kann er ihn dadurch auch aktivieren, und umgekehrt, man kann versuchen, jemanden für etwas zu gewinnen, erregt bei ihm jedoch

(1986, S. 168) Auch über die Grenzen der natürlichen Sprache hinweg können wir eine ähnliche Bipolartät verfolgen, etwa im Verhältnis von Kunststück, seinem Autor und dessen Bestrebungen einerseits und von dem kommunikativen Wert des Kunststücks und seiner Einwirkung auf den Rezipienten andererseits. In letzter Konsequenz ist immer der Adressat der maßgebende Deuter einer Mitteilung, wöbei er freilich auch unkritisch oder unkompetent (mangelhafte Bildung) und daher nicht objektiv und ungerecht sein kann oder aus anderen Gründen das Scheitern der Realisation einer kommunikativen Absicht herbeiführen kann.

9 Ein weiteres Argument für die Begründung der Annahme von der möglichen Existenz einer rezeptiven Komponente der kommunikativen Funktion?

10 Der Text wird hier im weitesten Sinne des Wortes als Resultat eines kommunikativen Prozesses, der kommunikativen Tätigkeit verstanden. Gemeint sind also auch mündlich geführte Dialoge.

11 Die rede ist eigentlich von der propositional-semantischen und kommunikativ-operationalen Potenz von Sprachmitteln und von der Funktionstransposition von sprachlichen Mitteln (auch Kommunikationsverfahren). (Vgl. STARKE 1985, S. $213 \mathrm{ff}$.)

12 Nach unserer Meinung kann direkte Bedeutung einer Mitteilung eine direkte Rezeption und eine begleitende (Neben)rezeption auslösen. Im 1. Fall geht es um das übliche Kommunikationsereignis, im 2. Fall um eine künstlerisch-ästhetische Wirkung, z. B. in einer künstlerischen Interpretation eines Textes: Des öfteren gehen aber u. E. die direkte und indirekte, begleitende Rezeption mit versteckter Bedeutung einer Mitteilung einher. Im ersten Fall ist somit eine Art Manipulation im Spiel, im zweiten dagegen das Entlarven. 
nur Widerwillen und Abneigung $u$. d. m. Die hier angeführten Kombinationen wir haben keine Vollständigkeit angestrebt - können auch unbewußt (also unbeabsichtigt) zum Vorschein kommen (vgl. oben zur rezeptiven Komponente der kommunikativen Funktion). Sehr wichtig ist bei den Erwägungen dieser Art jedenfalls auch die Beachtung gesellschaftlich-sozialer und ethnisch-kulturhistorischer und nicht zuletzt auch der damit $z$. T. korrelierenden sprachlichen Normen. Von Bedeutung ist also, daß der potentielle Adressat die Kriterien der Dekodierung (und nach Möglichkeit auch die der Kodierung ${ }^{13}$ ) von Kommunikationsabsichten kennt, mit anderen Worten, daß er in der Lage ist, die unmittelbare und die mögliche mittelbare kommunikative Funktion eines Textes $^{14}$, bzw. der in ihm verwendeten Ausdrucksmittel zu erfassen. Aus dem Gesagten geht hervor, daß bei der Betrachtung eines Kommunikationsprozesses auch die Rezeptionsphase an Bedeutung zunimmt. In der Tat hat sich in der Sprachwissenschaft der letzten Jahre die Tendenz bemerkbar gemacht, sich entsprechend auch auf den Rezeptionsprozeß zu konzentrieren, der auch nach bestimmten, jedoch nicht immer vorhersehbaren Gesetzmäßigkeiten verläuft und den gesamten Komplex der Faktoren und Bedingungen der sprachlichen Kommunikation wesentlich mitbestimmt. ${ }^{15}$ Die Textfunktion ist demzufolge nicht nur seine Zielgerichtetheit bzw. seine Zweckbestimmtheit (im Rahmen übergeordneter Tätigkeit), sondern der Text hat auch für den Rezipienten eine Funktion, die mit dessen Erwartungen, Absichten und Interessen beim Herangehen an den Text übereinstimmen kann oder nicht. Davon hängt der Grad der Eignung bzw. der Nützlichkeit/Förderlichkeit von Texten ab, der aus der Perspektive des Rezipienten eingeschätzt wird und in letzter Konsequenz dazu führen kann, daß "ein ungeeigneter und dem Rezeptionsziel nicht dienlicher Text... im allgemeinen vom Rezipienten als Rezeptionsobjekt auch nicht akzeptiert /wird/“ (HEUSINGER 1987, S 195).

\section{SCHLUßWORT}

Die kommunikative Funktion ist ein Begriff, der in der Linguistik Synonym für verschiedentlich interpretierte Kategorien ist und war. Von den grundlegenden Lexikonbedeutungen des Wortes (Aufgabe, Abhängigkeit) ausgehend, hat sich über Zwischenphasen, meist unter dem Einfluß der funktionalen Grammatik (Funktion ist gleich Bedeutung, Inhalt, Wirkung, Effekt auf den Leser/Hörer), in der funktional-kommunikativen Sprachbeschreibung unter Beachtung des Tätigkeitscharakters der Komunikation die Definition von Wilhelm SCHMIDT herausgebildet und durchgesetzt:

"Die Zielgerichtetheit von Tätigkeiten und ihrer konkreten Realisierungen, nämlich der Handlungen mit ihren Operationen und zugleich die Zweckbe-

13 Nach HARTUNG (1977, S. 14) müsse der Rezipient über das gleiche Wissen über Regelmäßigkeiten verfügen wie der Produzent.

14 HEUSINGER (1987, S. 194) spricht von “speziellen Intentionen, die der grundlegenden, im Text sich sprachlich ausprägenden Absicht untergeordnet sind“.

15 Vgl. MICHEL 1986, HEUSINGER 1987 und HEUSINGER 1987 a. 
stimmtheit der Instrumente/Mittel von Tätigkeiten, Handlungen und Operationen nennen wir ihre Funktion“ (SCHMIDT 1982, S. 15 f.).

Darüber hinaus wird auf weitere zu beachtende Momente hingewiesen, die bei der Behandlung des Begriffs der kommunikativen Funktion nicht außer acht gelassen werden dürften, so z. B. auf die sog. rezeptive Komponente des Funktionsbegriffs und auf die Tatsche, daß der Text auch eine Funktion für den Rezipienten hat. Außerdem werden hervorgehoben die Möglichkeit einer Unterscheidung von unmittelbarer und mittelbarer Ausprägung der kommunikativen Funktion sowie die Annahme, daß im Rahmen übergeordneter. Tätigkeiten auch der Textfunktion i. w. S. der Charakter der Zweckdienlichkeit zukommen kann.

\section{AUSWAHLBIBLIOGRAPHIE}

Bieberle, Bruno (1987): Zum Erschließen des Sinns von Texten. In: Deutschunterricht, 40, (1987), H. 4, S. 198-203.

Dimter, Matthias (1981): Textklassenkonzepte heutiger Alltagssprache. Max Niemayer Tübingen.

Hannappel, H./Melenk, H. (1979): Alltagssprache. Semantische Grundbegriffe und Analysebeispiele. Wilhelm Fink München.

Hartung, Wolfdietrich u. Autorenkollektiv (1976)2: Sprachliche Kommunikation und Gesellschaft. Akademie-Verlag Berlin.

Hartung, Wolfdietrich u. Autorenkollektiv (1977): Normen in der sprachlichen Kommunikation. Akademie-Verlag Berlin.

Helbig, Gerhard (1968): Zum Funktionsbegriff in der modernen Linguistik. In: Deutsch als Fremdsprache 5, (1968), $274 \mathrm{ff}$.'

Heusinger, Siegfried (1987): Zur Tiefe des Verstehens. In: Deutschunterricht 40 (1987), H. 4., S. 193-198.

Heusinger, Siegfried (1987): Zum Erfassen der Textfunktion durch den Rezipienten. In: Wissenschaftliche Zeitschrift der PH Potsdam, H. 5, Potsdam (in Druck) (1987a).

Langner, Helmut (1982): Zu einigen Grundpositionen sprachlicher Veränderungen. In: Beiträge zur Erforschung der deutschen Sprache. 2. Bd., Leipzig, S. 204 $-217$.

Michel, Georg (1983): Buchbesprechung von "Alltagssprache" (von Hannappel/Melenk). In: Zeitschrifft für Germanistik, H. 4 (1983), S. 469-472.

Michel, Georg (1986): Textsemantik, Textfunktion und Textstil. In: Potsdamer Forschungen, R. A., Heft 82, Potsdam 1986, S. 67.

Philosophisches Wörterbuch (1976), hrsg. von G. Klaus und M. Buhr, 2 Bde., 12. Aufl., Leipzig.

Schmidt, Wilhelm und Autorenkollektiv (1981): Funktional-kommunikative Sprachbeschreibung. Theoretisch-methodische Grundlegung, Bibliographisches Institut, Leipzig. 
Schmidt, Wilhelm (1982): Zum Funktionsbegriff in der neueren Linguistik, insbesondere in der funktional-kommunikativen Sprachbeschreibung. In: ZPSK, H. 1, Bd. 35 (1982), S. 9-18.

Schmidt, Wilhelm (1982): Zu einigen methodologischen Fragen einer kommunikationsorientierten wissenschaftlichen Sprach- und Textbeschreibung. In: Linguistiche Studien A, 99, Berlin, S. 1-10, (1982a).

Starke, Günter (1985): Probleme der funktional-kommunikativen Sprachbeschreibung. In: Zeitschrift für Germanistik, H. 2 (1985), S. 213-215.

Thiele, Rudolf (1983): Buchbesprechung von "Textklassenkonzepte heutiger Alltagssprache" (von Dimter). In: Zeitschrift für Germanistik, H. 1 (1983), S. 98-101.

Wörterbuch der deutschen Gegenwartssprache (1967), hrsg. von R. Klappenbach und W. Steinitz, 2. Bd., Berlin (WDG).

Povzetek

K POJMU KOMUNIKATIVNE FUNKCIJE V JEZIKOSLOVJU

Komunikativna funkcija je pojem, $\mathrm{ki}$ je $\mathrm{v}$ lingvistiki rabljen kot sinonim za različno interpretirane kategorije. Izhajajoč iz temeljnih slovarskih pomenov besede funkcija (naloga, odvisnost), se je preko vmesnih faz, največ pod vplivom funkcionalne slovnice ( $\mathrm{ki}$ funkcijo istoveti s pomenom, vsebino, učinkom na bralca/poslušalca) v funkcionalno-komunikativnem opisu jezika ob upoštevanju dejavnostnega značaja komunikacije izoblikovala in uveljavila SCHMIDTova definicija komunikativne funkcije:

“Ciljna naravnanost dejavnosti in njih konkretnih realizacij, namreč dejanj z njihovimi operacijami, ter hkrati smotrnost instrumentov/sredstev dejavnosti, dejanj in operacij imenujemo njihovo funkcijo“" (SCHMIDT 1982, S. 15 f).

Poleg tega je v prispevku govor o drugih pomembnih vidikih komunikativne funkcije, takon. pr. o t. i. receptivni komponenti v pojmu komunikativne funkcije in o dejstvu, da ima besedilo tudi funkcijo za recipienta. Razen tega je poudarjena možnost razlikovanja neposrednosti in posrednosti komunikativne funkcije kakor tudi podmena, da ima lahko $v$ okviru nadrejenih dejavnosti tudi besedilna funkcija $v$ šiřem smislu značaj smotrnosti. 